\title{
Metabolic responses of acute phase protein, antioxidant and cytokine in Arabian endurance horses.
}

\begin{abstract}
Changes in serum amyloid A (SAA), glutathione reductase (GR) and interleukin-6 (IL-6) could be responsible for the metabolic disorders and poor performance in Arabian horses during gruelling endurance events. Therefore, this study aims to evaluate the metabolic responses in relation to SAA, GR and IL-6 in Arabian horses during endurance events. After physical examination, the successfully completed (SC) $(n=16)$ and eliminated-from-race (ER) $(n=16)$ endurance horses were identified. The data obtained were analysed using ANOVA and pairwise correlations and the analysis were considered significant at $\mathrm{P}<0.05$. There were significant differences between the SC and ER Arabian horses in haemoglobin $(\mathrm{Hb})$, packed cell volume (PCV), creatine kinase, plasma protein, lactate and monocytes $(\mathrm{P}<0.0001)$, IL-6, GR $(\mathrm{P}<0.0007)$, SAA $(\mathrm{P}<0.0068)$, glucose $(\mathrm{P}<0.00342)$, aspartate transaminase $(\mathrm{P}<0.0049)$ concentrations. There were significant negative correlations between GR and plasma protein $(\mathrm{r}=-0.2830 ; \mathrm{P}<0.0299)$ and $\mathrm{GR}$ and SAA $(\mathrm{r}=-0.2554 ; \mathrm{P}<0.0489)$. There were significant positive correlations between IL-6 and $\mathrm{Hb}(\mathrm{r}=0.2977 ; \mathrm{P}<0.0220)$ and positive and not significant correlation between IL-6 and PCV ( $\mathrm{r}=0.2449 ; \mathrm{P}<0.0615)$. In conclusion, SAA, GR and IL-6 were significantly associated with metabolic crises in Arabian endurance horses. Therefore, SAA, GR and IL-6 could be used to evaluate performance and health status of endurance horses during training and endurance events.
\end{abstract}

Keyword: Performance; Glutathione reductase; Serum amyloid A; Interleukin 6. 